\title{
Estudio piloto de variables socio-emocionales, ansiedad y flow en alumnos de grado profesional de música mediante actividades BAPNE
}

\section{Pilot study of socio-emotional variables, anxiety and flow in music students through BAPNE activities}

\author{
Laura Moral Bofill ${ }^{1 *}$ \\ lauramoralbofill@gmail.com \\ Francisco Vicedo Molla** \\ pacovicedo@gmail.com \\ Francisco Javier Romero Naranjo*** \\ bapne.central@gmail.com \\ * Universidad Nacional de Educación a Distancia, UNED, España \\ **Conservatorio de Música y Danza Profesional de Palma de Mallorca, España \\ *** Universidad de Alicante, España
}

\section{Resumen:}

El objetivo de esta investigación es hacer un análisis exploratorio a través de un diseño cuasi-experimental con grupo control no equivalente de los efectos que las actividades del método BAPNE tienen sobre las emociones, la ansiedad, el "flow" y la conducta social en alumnos de Grado Profesional de Música. Los resultados obtenidos apoyan nuestras hipótesis de investigación con diferencias estadísticamente significativas en medidas pre-post del grupo experimental con $\mathrm{p}<.05$ para emociones, ansiedad-estado y flow. Sugiriendo que las actividades BAPNE, favorecen las emociones agradables, disminuyen la ansiedad estado y generan una

\begin{abstract}
:
Adopting a quasi-experimental design with a non-equivalent control group, this study conducts an exploratory analysis of the effects that BAPNE activities have on the emotions, anxiety, flow, and social behavior of music students. The results obtained support our research hypothesis with statistically significant differences in pre-post measures of the experimental group with $p<.05$ for emotions, stateanxiety and flow. They suggest that BAPNE activities favor pleasant emotions, decrease state anxiety and generate a high flow experience. For social behavior, however, no significant changes were reported for the measures taken with the BAS3 ques-
\end{abstract}

1 Dirección para correspondencia (correspondence address):

Laura Moral Bofill. C/Juan del Rosal, 10. 28040, Madrid (España). 
Estudio piloto de variables socio-emocionales, ansiedad y flow en alumnos de grado profesional de música mediante actividades BAPNE

Laura Moral Bofill, Francisco Vicedo Molla y Francisco Javier Romero Naranjo

experiencia alta de flow. Para la conducta social, sin embargo, no se han detectado cambios significativos en las medidas tomadas con el cuestionario BAS3.

\section{Palabras clave:}

Ansiedad; BAPNE; emociones; flow; educación musical; socialización. tionnaire.

\section{Key words:}

Anxiety; BAPNE; emotions; flow; music education; socialization.

\section{Resumé:}

Adoptant un design quasi-expérimental avec un groupe témoin non-équivalent, cette recherche conduit une analyse exploratoire des effets que les activités de la méthode BAPNE ont sur les émotions, l'anxiété, le "flow" et le comportement social chez les étudiants en musique. Les résultats obtenus soutiennent notre hypothèse de recherche avec des différences statistiquement significatives dans les mesures pré-post du groupe expérimental avec $\mathrm{p}<.05$ pour les émotions, I'anxiété état et le flow. Ils suggèrent que les activités de BAPNE favorisent les émotions agréables, diminuent l'anxiété état et génèrent une expérience élevée de flow. Pour le comportement social, cependant, aucun changement significatif n'a été détecté dans les mesures prises avec le questionnaire BAS3.

\section{Mots-clés:}

Anxiété; BAPNE; émotions; flow; éducation musicale; socialization.

Fecha de recepción: 11-3-2018

Fecha de aceptación: 18-11-2018

\section{Introducción}

Actualmente sabemos que el aprendizaje socioemocional (ASE) es de importancia vital para el desarrollo psicosocial y es un pre-requisito para un óptimo aprendizaje. Hay un gran volumen de investigaciones en el ámbito de la educación infantil y obligatoria que analizan la necesidad de desarrollar habilidades ASE y cómo se relaciona con indicadores emocionales, sociales y académicos. Moral-Bofill, Romero-Naranjo, Albiar-Aliaga y Cid-Lamas (2015) exponen, por un lado, que los resultados de estas investigaciones muestran cómo el rendimiento académico de los estudiantes aumenta y se relaciona con resultados positivos en el ámbito personal y social. Por otro lado, cómo el clima escolar contribuye, o no, a que los alumnos establezcan lazos de confianza, pertenencia, seguridad (necesidades psicológicas básicas), con los contextos educativos, ya que los aspectos afectivos individuales están influenciados y, a su vez, van a influir en la percepción del clima global del centro. Un campo menos explorado en este sentido son los centros educativos especializados de música, donde la mayoría de estudios se centran en el tema de la 
ansiedad, concretamente de la ansiedad escénica y sus relaciones con diferentes variables.

De igual manera, las investigaciones en neurociencia (Bueno, 2017; Márquez \& Tresserra, 2018; Mora, 2017) están aportando en los últimos años información de cómo funciona el cerebro de cara a mejorar los procesos y estrategias de enseñanza-aprendizaje y están dando un apoyo científico bien fundamentado a la necesidad de introducir elementos socioemocionales en educación.

Ambas líneas de investigación dan pistas para evaluar aspectos del funcionamiento en los centros de enseñanza desde diferentes niveles de aproximación: objetivos y contenidos de los currículos, estrategias de enseñanza-aprendizaje, tipo de funcionamiento del centro definido por la cultura de centro, valores, organización, interrelaciones que se establecen entre todos las personas, clima psicosocial, etc.

BAPNE es un método de estimulación cognitiva, socio-emocional, psicomotriz y rehabilitativo. Aunque el método no se define como método musical, trabaja con música y a través de la música. La investigación etnomusicológica realizada por su autor, Francisco-Javier Romero Naranjo, se centra en el uso del cuerpo como instrumento, y lo ha llevado a hacer un extenso estudio (Romero-Naranjo, 2013) y recopilación de materiales de diferentes culturas, especialmente de África. Con la aportación de diferentes disciplinas que son las que forman el acrónimo BAPNE (Biomecánica, Anatomía, Psicología, Neurociencia, Etnomusicología) diseña actividades donde el ritmo, el movimiento, la percusión corporal, el canto y la medida son el eje central, y su principal objetivo estimular aspectos cognitivos, socioemocionales y psicomotrices (Romero-Naranjo, 2017a, 2018). Todo ello bajo el paradigma de la teoría de las Inteligencias Múltiples (IM) de Howard Gardner (Gardner, 1987, 1995, 2010), y con especial atención al argumento que cada inteligencia se desarrolla mejor si se comprende con qué otras capacidades está relacionada y, por tanto, si se trabaja de manera más transversal.

Moral-Bofill et al. (2015) analizan la capacidad del método BAPNE para desarrollar habilidades ASE y sugieren que el método estimula las emociones, especialmente emociones agradables. Observan que son actividades que generan un nivel de flow importante, aportando bienestar psicofísico, repercutiendo en el control ejecutivo y favoreciendo el aprendizaje. También sugieren que puede estimular la sociabilidad y la empatía, y que puede ayudar a mejorar el clima del centro donde se 
aplique. Nosotros hemos realizado un estudio cuantitativo para medir algunas de estas variables, como son: las emociones, la ansiedad, el flow y la conducta social.

Por otro lado, los alumnos de música son un grupo de población que se puede beneficiar de manera doble con el aprendizaje de las actividades BAPNE, tanto en los aspectos que pretende estimular (cognitivos, socioemocionales, psicomotrices) como de manera específica, por los contenidos musicales vinculados a su aprendizaje.

\section{Emociones}

Para abordar el tema de las emociones hemos utilizado el modelo "Universos Emocionales" del psicólogo Roberto Aguado (Aguado, 2014). Como dice Aguado (2014), hay dos escenarios muy distintos desde donde el cerebro procesa la información: el escenario de la razón y el cerebro vincular. El cerebro vincular analiza la realidad desde el constante de si lo que ocurre es peligroso o no y si estamos en un momento de seguridad vital o no. Su propósito es la adaptación y ser capaces de crear reacciones que nos ayuden a enfrentarnos a las situaciones, de manera que lo que hacemos está condicionado por la emoción que se activa con la situación vivida y no tanto por la información que entendemos o comprendemos desde la razón.

De cara al aprendizaje y para el bienestar personal y social de los alumnos, las emociones agradables son las que van a movilizar el fondo emocional propicio para una vinculación significativa con el contexto educativo en general y los aprendizajes en particular. Las emociones agradables para Aguado (2014) serían: Curiosidad, Admiración, Seguridad y Alegría. Las emociones desagradables serían: Tristeza, Rabia, Asco, Culpa, Miedo. Y propone una emoción mixta: Sorpresa.

\section{Ansiedad}

Un referente básico para la mayoría de estudios sobre la ansiedad y que nosotros también retomamos es la teoría que desarrolla Spielberger, la cual se centra en las relaciones existentes entre "estado-rasgo-proceso" (Spielberger, 1966; Spielberger, Gorsuch \& Lushene, 1970; Spielberger, 1972a, 1972b). Para Spielberger la ansiedad es un proceso, un continuo de cogniciones, afectos y conductas provocadas por alguna forma 
de estrés. Si se interpreta como peligroso, aumenta la ansiedad-estado con todo el proceso de activación fisiológica relacionado. Entonces la ansiedad se intentará disminuir desde diferentes formas, reinterpretando la situación/estímulo, evitándola, con conductas más o menos adaptativas. Por otro lado, la aparición e intensidad de la ansiedad-estado (A-E) estará relacionada con características de cada persona, es decir, con la ansiedad-rasgo (A-R), entendida como las diferencias individuales estables de propensión a la ansiedad.

Si a través de actividades que contemplen el ASE disminuimos la A-E vinculada al contexto educativo, puede ayudar a mejorar el aprendizaje, $y$, si es suficientemente consistente y regular, tal vez modificará la A-R de los alumnos a niveles más óptimos.

\section{Flow}

Respecto al concepto de flow, su creador, Mihalyi Csikszentmihalyi (Csikszentmihalyi, 1975), lo define como un sentimiento de completa armonía, absorción total con la actividad realizada de forma tal que la motivación intrínseca y el placer de realizarla es la principal fuerza que dirige la participación. Por otro lado, Csikszentmihalyi (1998) expone que las actividades que inducen los estados de flow pueden llamarse "actividades de flow", porque hacen más probable que se produzca la experiencia permitiendo que una persona se concentre en objetivos que son claros y compatibles entre sí, a la vez que proporcionan una retroalimentación inmediata, dejando muy claro hasta qué punto una persona está actuando perfectamente. Lo que convierte las experiencias de flow en imanes para aprender, es decir, para desarrollar nuevos niveles de desafíos y habilidades. Csikszentmihalyi (1998), también habla de la personalidad autotélica. La persona autotélica haría las cosas por sí mismas, en lugar de hacerlas para conseguir después un objetivo externo. Siente que la mayoría de cosas que hace son importantes y válidas por sí mismas.

En el campo de la música hay estudios que relacionan flow con el aprendizaje musical en la línea que comentábamos aquí arriba, como imán para aprender, para involucrarse y permanecer en la actividad (Custodero, 1998, 2002, 2005). Nosotros pretendemos seguir esta línea y observar en qué medida la práctica de las actividades BAPNE en la educación musical profesional puede estimular la experiencia de flow. 


\section{Conducta social}

Las dinámicas y estrategias cooperativas e interactivas, como las propuestas en las actividades BAPNE, creemos que pueden movilizar aspectos facilitadores de la socialización, y, después de una intervención suficientemente larga, tal vez puedan generarse nuevas pautas de comportamiento. Un estudio empírico reciente de intervención con el método BAPNE es el de Fabra-Brell y Romero-Naranjo (2017). Con un diseño cuasi-experimental con grupo control han medido el grado de rechazo social de cada individuo de un grupo escolar. Los resultados sugieren que se elimina la competitividad entre los miembros del grupo y observan cambios para mejor en el grupo experimental en la medida postest.

Para seguir esta línea de estudio nosotros nos hemos centrado en las investigaciones realizadas por Silva y Martorell (1982, 1983) y Silva, Martorell y Clemente (1985) que han construido un conjunto de instrumentos de socialización. En concreto BAS-3 que indaga en la percepción que los propios sujetos tienen de su conducta social permitiendo lograr un perfil en función de cinco dimensiones: 1. Consideración con los demás (Co), sensibilidad social, preocupación por los demás, etc.; 2. Autocontrol en las relaciones sociales (Ac), convivencia en el mutuo respeto, acatamiento de reglas, etc. en el polo positivo y a la inversa en el negativo; 3. Retraimiento social (Re), apartamiento pasivo y activo de los demás, Ilegando al aislamiento; 4. Ansiedad social/timidez (At), diferentes manifestaciones de ansiedad y reacciones de timidez en las relaciones sociales; 5. Liderazgo (Li), popularidad, confianza en sí mismo, espíritu de servicio. A su vez se pueden agrupar como facilitadoras de la socialización o inhibidoras.

\section{Objetivos}

El objetivo de este estudio fue analizar a través de la intervención con el método BAPNE, los efectos de las actividades BAPNE sobre el estado emocional, la ansiedad y el estado de flow durante las sesiones, así como observar posibles cambios en aspectos facilitadores e inhibidores de la socialización de los alumnos.

Las hipótesis planteadas son las siguientes: 
1. La intervención a través de las actividades BAPNE incrementará las emociones agradables y disminuirá las desagradables.

2. La intervención a través de las actividades BAPNE disminuirá la ansiedad estado.

3. La intervención a través de las actividades BAPNE provocará un alto estado de flow.

4. La intervención a través de las actividades BAPNE mejorará factores facilitadores de la socialización.

\section{Método}

\section{Participantes}

En este estudio participaron 28 alumnos de Lenguaje Musical de $1 \mathrm{r}$ curso de Grado Profesional de Música, con edades comprendidas entre los 11 y los 17 años, a excepción de un joven-adulto de 27, con una media de $m=13,53$. Entre los participantes 22 eran chicas y 6 eran chicos, de clase social media, pertenecientes a dos Conservatorios Profesionales de Música Municipales de Cataluña y Baleares. Los alumnos son dos grupos naturales de cada conservatorio, de manera que uno ha sido el grupo experimental (GE) con $\mathrm{N}=14$ (12 chicas y 2 chicos) y otro el grupo control (GC) con $\mathrm{N}=14$ (10 chicas y 4 chicos). Todos los alumnos que empezaron la investigación finalizaron el estudio, pero un alumno del GC no pasó los postest y para el análisis el GC ha quedado en $\mathrm{N}=$ 13 (9 chicas y 4 chicos). Para algún cuestionario, tuvimos que eliminar algún caso por no haberse cumplimentado correctamente o porque no se correspondía con la edad del cuestionario.

\section{Instrumentos}

\section{Cuestionario Universos Emocionales (UE)}

Para medir los factores emocionales nos interesaba detectar qué emociones aparecen y se hacen más presentes durante las actividades. Dada la dificultad de medición y dada la escasez de instrumentos en esta línea, optamos por elaborar un cuestionario exploratorio basado en el modelo del psicólogo clínico Roberto Aguado (Aguado, 2014), Universos Emocionales. Estas dimensiones o universos los agrupa en: 
Estudio piloto de variables socio-emocionales, ansiedad y flow en alumnos de grado profesional de música mediante actividades BAPNE

Laura Moral Bofill, Francisco Vicedo Molla y Francisco Javier Romero Naranjo

1. Emociones agradables: Curiosidad, Admiración, Seguridad, Alegría (serán CASA).

2. Emociones mixtas: Sorpresa.

3. Emociones desagradables: Tristeza, Rabia, Asco, Culpa, Miedo (serán TRACM).

El cuestionario Universos Emocionales es, entonces, un reflejo de este modelo (tabla 1).

Tabla 1

Dimensiones del cuestionario Universos Emocionales.

\begin{tabular}{ll}
\hline ADMIRACIÓN & $\begin{array}{l}\text { tranquilidad, respeto, identificación, admiración, imitación, } \\
\text { fascinación }\end{array}$ \\
ALEGRÍA & $\begin{array}{l}\text { diversión, gratificación, contento, alegría, placer, satisfac- } \\
\text { ción, entusiasmo, euforia }\end{array}$ \\
CURIOSIDAD & $\begin{array}{l}\text { atracción, voluntad, expectación, curiosidad, interés, atre- } \\
\text { vimiento, arranque }\end{array}$ \\
SEGURIDAD & $\begin{array}{l}\text { serenidad, corrección, calma, seguridad, paz, control, } \\
\text { enraizar }\end{array}$ \\
TRISTEZA & $\begin{array}{l}\text { aburrimiento, desgana, frustración, tristeza, impotencia, } \\
\text { indefensión, dolor }\end{array}$ \\
RABIA & $\begin{array}{l}\text { enfado, irritabilidad, hostilidad, vergüenza, rabia, envidia, } \\
\text { celos, ira, odio, violencia }\end{array}$ \\
MIEDO & $\begin{array}{l}\text { tensión, ansiedad, angustia, miedo, horror, pánico, terror } \\
\text { ASCO }\end{array}$ \\
desagrado, menosprecio, rechazo, asco, repulsión, tirria \\
falta, error, imperfección, tropiezo, culpa, pudor, rubor \\
SORPRESA
\end{tabular}

En el cuestionario hay tres categorías de respuesta: ausencia de la emoción $=0$; presencia $=1$; permanencia $=2$. Más emociones marcadas en una dimensión, sugiere que el sujeto transita más por esa dimensión, tanto en matiz como a nivel de permanencia. De manera que a mayor puntuación en la suma de emociones elegidas por los sujetos y que formen parte de un mismo universo, se presupone mayor presencia y permanencia de esa dimensión.

STAIC

El cuestionario STAIC es un cuestionario de autoevaluación de la Ansiedad Estado/Rasgo en niños/adolescentes (9 a 15 años). Hemos utilizado 
la adaptación española por el Departamento de I+D de TEA Ediciones (Spielberger, 1989) del cuestionario STAIC, State-Trait Anxiety Inventory for Children (Spielberger et al., 1970). Tiene dos escalas, una para la medida de Ansiedad Estado (A-E) y otra para la Ansiedad Rasgo (A-R). Cada una de ellas contiene 20 elementos con los que el sujeto puede expresar cómo se siente. En el caso de A-E, cómo se siente en un momento determinado y en el caso de A-R, cómo se siente en general. La medida de A-E intenta apreciar estados transitorios de ansiedad (aprensión, tensión, preocupación, etc.) que fluctúan y varían en intensidad con el tiempo, mientras que la A-R, intenta evaluar diferencias relativamente estables de propensión a la ansiedad. Se han obtenido valores alfa de Cronbach en la toma pre y post respectivamente de STAIC para A-E de .818 y .840; y para A-R de .814 y .843.

\section{Escala de Flow disposicional (DFS) y Escala de Estado de Flow (FSS)}

El flow disposicional (DFS) refleja con qué frecuencia un sujeto experimenta flow cuando realiza una actividad elegida por él mismo e informando de manera retrospectiva. El flow estado (FSS) refleja el estado de flow durante una actividad y el cuestionario se realiza justo después de la misma. Utilizamos la versión traducida al español de la Dispositional Flow Scale (Jackson, Kimiecik, Ford \& Marsh, 1998) y, de la Flow State Scale (Jackson \& Marsh, 1996) realizada por Cervelló, Fenoll, Jiménez, García-Calvo y Santos-Rosa (2001) y validadas por García-Calvo, CerveIló, Jiménez y Santos-Rosa (2005) y más recientemente para el FSS por García-Calvo, Jiménez, Santos-Rosa, Reina y Cervelló (2008). Las dos escalas constan de 36 ítems y se diferencian básicamente en el enunciado -de cara a medir el flow disposicional, o bien, el estado de flow después de una actividad- y también se diferencian en la respuesta. Utilizando una escala tipo Likert de 1 a 5, en el FSS, $1=$ totalmente en desacuerdo y $5=$ totalmente de acuerdo, y en el DFS, $1=$ nunca y $5=$ siempre. Las dos escalas permiten obtener una puntuación global de flow a través de las medidas de sus nueve dimensiones: Equilibrio habilidad-reto; Fusión de acción-pensamiento; Objetivos claros; Feedback claro; Concentración en la tarea; Sentido de control; Pérdida de autoconciencia; Transformación del tiempo; Experiencia autotélica. El DFS obtuvo un valor alfa de Cronbach aceptable de .68 y el FSS excelente, de .95. En el DFS, las nueve dimensiones por separado dieron los siguientes índices de consistencia: Equilibrio habilidad-reto -.02; Fusión de acción-pensamiento .46; 
Objetivos claros .91; Feedback claro .57; Concentración en la tarea .79; Sentido de control .62; Pérdida de autoconciencia .83; Transformación del tiempo .32; Experiencia autotélica .87. Las escalas 1, 2 y 8, muestran índices inaceptables de consistencia interna y si las eliminamos, el factor global de flow para el DFS es, aunque no varía, de .68. Aspectos que tendremos en cuenta de cara a los análisis posteriores. En el FSS, las nueve dimensiones por separado dieron unos índices de consistencia de aceptables a excelentes: Equilibrio habilidad-reto .89; Fusión de acciónpensamiento .73; Objetivos claros .90; Feedback claro .88; Concentración en la tarea .70; Sentido de control .86; Pérdida de autoconciencia .98; Transformación del tiempo .95; Experiencia autotélica .89.

\section{BAS-3}

BAS-3 es una Batería de Socialización de Silva y Martorell (2009) que indaga la percepción que los propios niños y adolescentes tienen de su conducta social. Permite lograr un perfil de la conducta social en función de 5 dimensiones que se corresponden con las siguientes subescalas: Consideración con los demás (Co), con 14 ítems; Autocontrol en las relaciones sociales (Ac), con 14 ítems; Retraimiento social (Re), con 14 ítems; Ansiedad social/timidez (At), con 12 ítems; Liderazgo (Li), con 12 ítems y una escala de Sinceridad (S), con 10 ítems. Los autores encontraron índices de consistencia interna, con $\mathrm{N}=806$, de aceptables a buenos (coeficiente alfa .82, .78, .81, .78, .73, .60 respectivamente), nosotros con una muestra pequeña, $\mathrm{N}=26$, hemos obtenido los siguientes valores en el pretest: Co .46, Ac .54, Re .79, At .55, Li .56 y S .67. Y los siguientes en el postest: Co .65, Ac .79, Re .38, At .19, Li .68 y S .64. De manera que los análisis estadísticos posteriores se deben interpretar con cautela, a pesar de que los resultados, como veremos más adelante, son muy coherentes.

\section{Diseño, procedimiento y análisis}

El diseño utilizado es un diseño cuasi-experimental con grupo control no equivalente (Campbell \& Stanley, 1966) al utilizar dos grupos naturales de dos clases de Lenguaje Musical de dos Conservatorios Profesionales de Música.

El primer paso fue presentar la propuesta de investigación al equipo directivo de los dos conservatorios. Seguidamente se informó a los 
padres y recogimos los consentimientos firmados de los alumnos que participaron en el estudio.

Los dos grupos hacían dos horas semanales de clase de Lenguaje Musical. Las clases de Lenguaje Musical están enfocadas a formar el oído a través de todos los elementos musicales implicados (rítmico, melódico, armónico, formal, estético, conceptual, etc.).

El GE pasó la intervención, de manera que dedicó una hora a la semana a hacer actividades BAPNE (Romero-Naranjo, 2017b) adecuadas al nivel de los alumnos y con contenidos vinculados a las clases de Lenguaje Musical. Por su lado, el GC no pasó la intervención y siguió con sus clases habituales de Lenguaje Musical. La intervención se realizó entre los meses de marzo a mayo de 2017, con un total de 10 sesiones de 1 hora de duración a frecuencia de 1 hora semanal.

Antes de la intervención ambos grupos pasaron los diferentes cuestionarios descritos en el apartado Instrumentos, excepto el test de flow que solo lo pasó el GE. La razón por la que consideramos que sólo lo pasara el GE fue la convicción de que una clase tradicional de $1^{\circ}$ de Lenguaje Musical de Grado Profesional no es el tipo de actividad en la que esperes estimular el estado de flow, por lo que decidimos evitar hacer pasar un cuestionario más a los alumnos del GC. Entonces, en lugar de comparar el estado de flow entre grupos, pensamos que era más apropiado hacer una comparación intragrupo. Es por ello que el GE pasó la Escala de Flow Disposicional (DFS), la cual refleja con qué frecuencia un sujeto experimenta flow cuando realiza una actividad elegida por él mismo e informando de manera retrospectiva, así que pedimos a los alumnos del GE que pensaran en alguna actividad que implicara movimiento (deporte, danza, etc.) y que hubieran elegido ellos mismos. Lo pasaron como pretest para obtener información sobre la experiencia de flow que pudieran haber experimentado en otras actividades y que después nos permitiera comparar con el estado de flow en el FSS después de las sesiones BAPNE.

Inmediatamente después empezaron las sesiones para el GE de la mano de un formador-investigador del método BAPNE músico de profesión, psicólogo y especialista en Lenguaje Musical. Finalizada la intervención se volvieron a pasar los cuestionarios a los dos grupos, experimental y control, para analizar los posibles cambios producidos. Para todos los cuestionarios nos aseguramos que los alumnos tenían claro qué les estábamos pidiendo y que comprendían bien la instrucciones y 
el procedimiento para cada uno de ellos. Para realizar la escala de A-E del STAIC en el pretest, pedimos a los alumnos que se situaran en el contexto de las clases de lenguaje musical del conservatorio, ya que el cuestionario permite concretar una situación o vivencia particular para contestar las declaraciones de esta escala. Para el postest, tenían la misma instrucción, pero el GE contemplaba, entonces, la hora semanal que había dedicado a las actividades BAPNE.

El análisis estadístico que hemos realizado es un análisis bajo presupuestos no paramétricos básicamente por haber realizado un muestreo de conveniencia y por ser una muestra pequeña.

\section{Resultados}

\section{Universos Emocionales (UE)}

Calculamos estadísticos descriptivos (Tabla 2). Comparamos las medias de cada dimensión y de cada grupo y las pasamos a porcentajes para facilitar la comparación.

Tabla 2

Estadísticos descriptivos de UE ( $M=$ media; DE= desviación estandar).

\begin{tabular}{lllllllll}
\hline & \multicolumn{4}{c}{$\mathrm{GE}$} & \multicolumn{5}{c}{$\mathrm{GC}$} \\
\cline { 2 - 9 } & $\mathrm{N}$ & $\mathrm{M}$ & $\mathrm{DE}$ & $\%$ & $\mathrm{~N}$ & $\mathrm{M}$ & $\mathrm{DE}$ & $\%$ \\
\hline admiración pre & 12 & 1.50 & 1.087 & 12.5 & 13 & 1.85 & 1.144 & 15.41 \\
admirac. post & 12 & 1.75 & 1.288 & 14.58 & 13 & 1.38 & 1.121 & 11.50 \\
alegría pre & 12 & 2.92 & 1.975 & 18.25 & 13 & 1.77 & 1.235 & 11.06 \\
alegría post & 12 & 5.17 & 2.480 & 32.31 & 13 & 1.46 & 1.561 & 9.12 \\
curiosidad pre & 12 & 2.00 & 1.706 & 14.28 & 13 & 1.31 & .630 & 9.35 \\
curiosidad post & 12 & 2.42 & 2.021 & 17.28 & 13 & 1.23 & .832 & 8.78 \\
seguridad pre & 12 & 2.33 & 1.775 & 16.64 & 13 & 2.08 & 2.290 & 14.85 \\
seguridad post & 12 & 2.67 & 1.923 & 19.07 & 13 & 1.23 & 1.235 & 8.78 \\
tristeza pre & 12 & 1.67 & 1.073 & 11.92 & 13 & 1.92 & 1.115 & 13.71 \\
tristeza post & 12 & .50 & .905 & 3.57 & 13 & 1.15 & .899 & 8.21 \\
rabia pre & 12 & .83 & .577 & 4.15 & 13 & .62 & .768 & 3.1 \\
rabia post & 12 & .42 & .515 & 2.1 & 13 & .46 & .660 & 2.3 \\
miedo pre & 12 & .92 & .515 & 6.57 & 13 & .62 & .650 & 4.42 \\
\hline miedo post & 12 & .58 & .669 & 4.14 & 13 & .69 & .855 & 4.92
\end{tabular}




\begin{tabular}{lllllllll}
\hline & \multicolumn{3}{c}{ GE } & \multicolumn{5}{c}{ GC } \\
\cline { 2 - 9 } & $\mathrm{N}$ & $\mathrm{M}$ & $\mathrm{DE}$ & $\%$ & $\mathrm{~N}$ & $\mathrm{M}$ & $\mathrm{DE}$ & $\%$ \\
\hline asco pre & 12 & .25 & .452 & 1.78 & 13 & .31 & .630 & 2.21 \\
asco post & 12 & .08 & .289 & 0.57 & 13 & .08 & .277 & 0.57 \\
culpa pre & 12 & 1.58 & 1.311 & 11.28 & 13 & 1.38 & 1.193 & 9.85 \\
culpa post & 12 & 1.08 & 1.084 & 7.71 & 13 & .69 & .751 & 4.92 \\
sorpresa pre & 12 & 1.58 & 1.311 & 15.8 & 13 & .77 & .725 & 7.7 \\
sorpresa post & 12 & 1.33 & 1.557 & 13.3 & 13 & .54 & .519 & 5.4 \\
CASA pre & 12 & 8.75 & 5.311 & 15.62 & 13 & 7.00 & 4.041 & 12.5 \\
CASA post & 12 & 12.00 & 5.954 & 21.42 & 13 & 5.23 & 3.419 & 9.33 \\
TRACM pre & 12 & 5.33 & 1.969 & 7.01 & 13 & 4.92 & 2.465 & 6.47 \\
TRACM post & 12 & 2.58 & 2.151 & 3.39 & 13 & 3.15 & 2.444 & 4.14 \\
\hline
\end{tabular}

La comparación de las medias pretest-postest en el GE muestra que ha habido un aumento de las dimensiones agradables, sobre todo de Alegría, que aumenta del $18.25 \%$ al $32.31 \%$. Y, a su vez, ha habido una disminución de todas las dimensiones desagradables, sobre todo de Tristeza, que disminuye del $11.92 \%$ al $3.57 \%$. En el GC, en cambio, han disminuido las agradables, sobre todo Seguridad, que disminuye del $14.85 \%$ al $8.78 \%$ y las dimensiones desagradables también disminuyen, excepto Miedo que aumenta ligeramente. Con la prueba $U$ de Mann-Whitney de comparación de dos muestras independientes comparamos los pretest del GE y el GC para ver la equivalencia inicial de los dos grupos, no mostrando diferencias estadísticamente significativas. Con la Prueba de los Rangos con Signo de Wilcoxon comparamos el pre y el post de todas las dimensiones y también para las dimensiones agrupadas como CASA y TRACM. En el GE hay diferencias estadísticamente significativas con $p<.05$ en: Alegría $(z=2.777$ $\mathrm{p}=.005) ;$ Tristeza $(z=-2.263 \mathrm{p}=.024) ; \operatorname{CASA}(z=2.143 \mathrm{p}=.032)$; $\operatorname{TRACM}(z=-2.564 p=.010$.) De manera que podemos decir que haciendo las actividades BAPNE se han generado emociones agradables $y$, a su vez, han disminuido las emociones desagradables $y$, que las diferencias, por tanto, entre el pretest y el postest no son debidas al azar. En el pretest-postest del GC no hay diferencias estadísticamente significativas excepto en Tristeza $(z=-2.058 \mathrm{p}=.040)$. Para el resto de dimensiones, ni para las agrupaciones CASA y TRACM, no ha habido cambio significativo en el GC.

En el GE la agrupación TRACM refleja como: aburrimiento, desgana; 
tensión, ansiedad; desagrado; error, imperfección; vergüenza; han disminuido. La agrupación CASA refleja, sobre todo, como ha aumentado la dimensión Alegría. En la dimensión Seguridad, que aumenta de manera discreta, los alumnos sentían más "corrección" en el pretest y, luego, en el postest, "corrección" disminuye y aumenta "paz". De manera que observamos un cambio cualitativo que a nivel cuantitativo no se percibe porque se compensan las puntuaciones. Creemos que "corrección" está más asociada a aspectos formales de comportamiento en las clases y, en cambio, el aumento de "paz" (como calma o serenidad) se relaciona más, y de ahí su aumento, a la disminución de la ansiedad estado durante las sesiones, como veremos más adelante. Para cerrar el análisis de UE realizamos, con los postest de los dos grupos, una comparación para muestras independientes con la prueba $U$ de Mann-Whitney, mostrando diferencias estadísticamente significativas para Alegría $(z=3.519$ $p=.000)$, CASA $(z=2.866 p=.004)$, Seguridad $(z=1.983 p=.047)$ y Tristeza con $p<.1(z=-1.903 p=.057)$.

\section{Ansiedad (STAIC)}

Calculamos estadísticos descriptivos (Tabla 3).

Tabla 3

Estadísticos descriptivos STAIC.

\begin{tabular}{lcccccc}
\hline & $\mathrm{N}$ & Media GE & DE & $\mathrm{N}$ & Media GC & DE \\
\hline A-E pre & 12 & 33.17 & 5.096 & 12 & 31.00 & 6.620 \\
A-E post & 12 & 29.08 & 4.621 & 12 & 32.08 & 7.428 \\
A-R pre & 12 & 34.75 & 5.610 & 12 & 36.25 & 6.956 \\
A-R post & 12 & 36.42 & 5.265 & 12 & 36.08 & 8.426 \\
\hline
\end{tabular}

La comparación de las medias pretest-postest del GE refleja una disminución de la A-E en el postest, es decir, los alumnos se sienten menos ansiosos, menos tensos. A-R, en cambio, se mantiene estable. Las medias pretest y postest del GC se mantienen estables tanto para A-E como para A-R. Con la prueba $U$ de Mann-Whitney de comparación de dos muestras independientes comparamos los pretest de los dos grupos, no mostrando diferencias estadísticamente significativas y estableciendo así su equivalencia inicial en ansiedad. Con la Prueba de los Rangos con Signo de Wilcoxon comparamos pre y post de cada grupo. 
El GE muestra diferencias estadísticamente significativas en el nivel de A-E antes y después de las sesiones $(z=-2.317 p=.020)$, por lo que podemos decir que las diferencias son efecto de la intervención. La escala de A-R, en cambio, no ha mostrado cambios estadísticamente significativos $(z=0.551 \mathrm{p}=.581)$. Por otro lado, el GC no ha mostrado diferencias estadísticamente significativas pre y post ni para A-E ni para A-R (A-E: $z=0.040 p=.968$ y A-R: $z=0.089 p=.929$ ). Para cerrar el análisis sobre la ansiedad, realizamos, con los postest de los dos grupos, una comparación para muestras independientes con la prueba U de Mann-Whitney, no mostrando diferencias estadísticamente significativas ni para A-E, ni para A-R. Aunque se percibe claramente en los postest de A-E una diferencia entre los dos grupos, la prueba no ha llegado a ser significativa $(z=-0.956 p=.347)$.

\section{FLOW (DFS, FSS)}

La información de los cuestionarios DFS y FSS presentan diferentes perspectivas de interpretación. Lo primero que hicimos fue calcular estadísticos descriptivos (Tabla 4).

Tabla 4

Estadísticos descriptivos Flow.

\begin{tabular}{lcccccc}
\hline & $\mathrm{N}$ & Media DFS & $\mathrm{DE}$ & $\mathrm{N}$ & Media FSS & $\mathrm{DE}$ \\
\hline Equilibrio H-R & 14 & 3.3786 & .39551 & 14 & 4.1250 & .70540 \\
Fusión A-P & 14 & 2.9107 & .52447 & 14 & 3.4286 & .87392 \\
Objetivos claros & 14 & 3.3571 & 1.03642 & 14 & 3.8214 & .76854 \\
Feedback claro & 14 & 3.5179 & .53227 & 14 & 4.1429 & .67734 \\
Concentr.-tarea & 14 & 3.3571 & .66299 & 14 & 3.6429 & .65570 \\
Sentido de control & 14 & 3.4107 & .53356 & 14 & 3.6071 & .65570 \\
Perdida de autoc. & 14 & 3.7321 & .99259 & 14 & 4.1786 & 1.03973 \\
Transf. tiempo & 14 & 3.4286 & .47463 & 14 & 3.3750 & .94944 \\
Exp. autotélica & 14 & 3.3571 & .80691 & 14 & 4.0714 & .78095 \\
FLOW GLOBAL & 14 & 3.3821 & .27150 & 14 & 3.8164 & .54791 \\
\hline
\end{tabular}

Si observamos las medias del DFS tanto del total de flow, como de cada dimensión, vemos como los alumnos informan de una experiencia de flow moderada, con puntuaciones alrededor de 3 (mín. $m=2.9107$ y máx. $m=3.7321$ ) y con una media total de flow de $m=3.3821$. Estos resultados sugieren que los alumnos reconocen una frecuencia mo- 
derada de la experiencia de flow para su actividad elegida y, por otro lado, sugieren, según una de las interpretaciones para este test (Jackson \& Eklund, 2004), que no estamos delante de un grupo de personalidades autotélicas. Las medias del FSS muestran que la experiencia de flow del grupo, durante la actividad BAPNE, ha fluctuado entre $\mathrm{m}=3.3750$ y $\mathrm{m}=$ 4.1786 para las diferentes dimensiones, con una media total de flow de $\mathrm{m}=3.8164$. Estos resultados sugieren que los alumnos han experimentado, durante las actividades BAPNE, una experiencia de flow alta $(>4)$, para: Equilibrio habilidad-reto, Feedback claro, Pérdida de autoconciencia y Experiencia autotélica. Moderada-alta $(>3,5)$ para: Objetivos claros, Concentración tarea y Sentido de control. Y moderada (>3) para: Transformación del tiempo.

Si conforme a los resultados del DFS, suponemos que no estamos delante de personalidades autotélicas, podemos suponer que es la vivencia de la actividad en sí misma la que ha hecho sentir un alto estado de flow o, dicho de otra manera, que es la actividad que es autotélica. Así que analizamos las diferencias entre los resultados del DFS y el FSS a través de la prueba para muestras relacionadas con Rangos de Wilcoxon obteniendo diferencias estadísticamente significativas entre los dos cuestionarios para: Feedback claro $(z=2.585 p$ $=.010)$; Pérdida de autoconciencia $(z=2.106 \mathrm{p}=.035)$; Experiencia autotélica $(z=2.331 \mathrm{p}=.020)$ y para el factor global de flow $(z=$ $2.668 \mathrm{p}=.008)$. Para el objetivo de nuestro estudio merece especial atención la dimensión "Experiencia Autotélica" con una $\mathrm{m}=4.07$ en el FSS y, por tanto, con una alta percepción de estado de flow para la actividad BAPNE, percibido como: disfrute de la experiencia, aporte de bienestar, sentimientos positivos al realizarla, gratificación y deseo de revivirla otra vez.

\section{BAS-3}

Calculamos estadísticos descriptivos (Tabla 5 y 6 ). 
Estudio piloto de variables socio-emocionales, ansiedad y flow en alumnos de grado profesional de música mediante actividades BAPNE laura Moral Bofill, Francisco Vicedo Molla y Francisco Javier Romero Naranjo

Tabla 5

Estadísticos descriptivos BAS-3, G.E.

\begin{tabular}{lccccc}
\hline & $\mathrm{N}$ & Media pre & $\mathrm{DE}$ & Media post & $\mathrm{DE}$ \\
\hline $\mathrm{Co}$ & 12 & 13.17 & 1.030 & 12.75 & 2.050 \\
$\mathrm{Ac}$ & 12 & 10.17 & 1.850 & 9.08 & 3.502 \\
$\mathrm{Re}$ & 12 & 2.50 & 3.371 & 1.42 & 1.730 \\
$\mathrm{At}$ & 12 & 3.42 & 2.503 & 3.42 & 2.234 \\
$\mathrm{Li}$ & 12 & 7.33 & 2.309 & 6.67 & 2.839 \\
$\mathrm{~S}$ & 12 & 6.17 & 2.290 & 5.25 & 2.340 \\
\hline
\end{tabular}

Tabla 6

Estadísticos descriptivos BAS-3, G.C.

\begin{tabular}{lccccc}
\hline & $\mathrm{N}$ & Media pre & $\mathrm{DE}$ & Media post & $\mathrm{DE}$ \\
\hline $\mathrm{Co}$ & 13 & 12.54 & 1.506 & 12.54 & 1.391 \\
$\mathrm{Ac}$ & 13 & 11.15 & 2.410 & 11.08 & 2.691 \\
$\mathrm{Re}$ & 13 & 1.85 & 1.864 & 1.38 & .961 \\
$\mathrm{At}$ & 13 & 4.23 & 1.964 & 3.69 & 2.463 \\
$\mathrm{Li}$ & 13 & 8.62 & 3.776 & 6.54 & 2.757 \\
$\mathrm{~S}$ & 13 & 4.54 & 2.295 & 4.92 & 2.216 \\
\hline
\end{tabular}

Las medias, tanto del GE como del GC, en el pretest, reflejan que la muestra total del estudio es muy homogénea en los patrones de comportamiento social que detectan las escalas. Son alumnos que presentan puntuaciones altas y moderadas en las escalas facilitadoras y puntuaciones bajas en las inhibidoras, lo cual es una buena indicación del nivel de socialización, así como de aspectos de personalidad que correlacionan con ellas, como son la empatía y la extraversión (Silva \& Martorell, 2009). Estas puntuaciones se mantienen estables en el postest. Con la prueba $U$ de Mann-Whitney de comparación de dos muestras independientes comparamos los pretest de los dos grupos no mostrando diferencias estadísticamente significativas en ninguna escala. Con la prueba para muestras relacionadas de Wilcoxon tampoco mostró diferencias estadísticamente significativas respecto pretest y postest en ninguno de los dos grupos. Para finalizar, con la prueba $U$ de Mann-Whitney de comparación de dos muestras independientes comparamos los postest de los dos grupos no mostrando diferencias estadísticamente significativas en ninguna escala. 


\section{Conclusiones}

Los resultados de este estudio piloto muestran diferencias significativas con $\mathrm{p}<.05$ en el pre-post del GE para las dimensiones emocionales, la ansiedad y el flow. Los datos de cada uno de los cuestionarios por separado confirman tres de las hipótesis planteadas: durante la intervención con las actividades BAPNE aumentarán las emociones agradables y disminuirán las desagradables, disminuirá la ansiedad estado y habrá una percepción alta de estado de flow. Los resultados muestran asociaciones directas o inversas (si disminuye la ansiedad, aumenta el flow y las emociones agradables, etc.).

En los resultados del STAIC para la escala de A-R, el GE no ha mostrado cambios estadísticamente significativos. Este resultado es esperable teniendo en cuenta la duración de la intervención, 10 sesiones de una hora, tiempo insuficiente para provocar cambios en la A-R.

Los resultados del BAS-3, los cuales no han mostrado diferencias estadísticamente significativas, son esperables teniendo en cuenta la duración del estudio que creemos que ha sido insuficiente para provocar cambios en los hábitos de funcionamiento social en general. Además, las puntuaciones del pretest ya indican un nivel de socialización alto, así que era difícil que aumentara más.

Los resultados sugieren que puede ser una línea de investigación educativa interesante a desarrollar ya que puede contribuir al bienestar del alumnado y al clima de centro, y, por tanto, a facilitar un buen desarrollo personal, social y académico. Además, en este caso concreto de los estudiantes de música, puede proporcionar un enfoque más global al tema de la ansiedad escénica. Pensamos que es una línea interesante de investigación estudiar la relación entre la A-E vinculada al contexto educativo, la A-R y la ansiedad escénica musical. Nos preguntamos si a través de una intervención regular y consistente podríamos conseguir disminuir la A-E y la A-R y que esto se asocie de manera directa con la disminución de la ansiedad escénica musical.

Para finalizar, se tiene que tener en cuenta las limitaciones del estudio. Es un estudio piloto con una muestra pequeña resultado de un muestreo de conveniencia. En cuanto el diseño creemos que es el más adecuado para este tipo de investigación con tratamiento y que es el que representa una menor amenaza contra la validez interna. Como ya hemos mencionado, hemos utilizado un análisis no paramétrico, el cual presupone que no es- 
Estudio piloto de variables socio-emocionales, ansiedad y flow en alumnos de grado profesional de música mediante actividades BAPNE laura Moral Bofill, Francisco Vicedo Molla y Francisco Javier Romero Naranjo

tamos haciendo inferencias poblacionales. Los resultados son válidos para el grupo experimental estudiado y están respaldados por los resultados que ha obtenido el GC. Ahora bien, no se pueden generalizar los resultados y esperamos poder realizar estudios longitudinales y con muestras mayores.

\section{Agradecimientos}

Agradecer al Conservatorio de Terrassa y al Conservatorio de Palma de Mallorca su colaboración, especialmente al Jefe de Estudios del Conservatorio de Terrassa, Dídac Moya, y, a su director, Jesús Silvestre, por habernos facilitado con tanta amabilidad y atención realizar esta investigación.

\section{Referencias}

Aguado, R. (2014). Es emocionante saber emocionarse. Madrid: EOS.

Bueno-Torrens, D. (2017). Neurociència per educadors. Barcelona: Rosa Sensat.

Campbell, D. T., \& Stanley, J. C. (1966). Experimental and Quasi-experimental Designs for Research. Nueva York: Rand McNally \& Company.

Cervelló, E. M., Fenoll, A. N., Jiménez, R., García-Calvo, T., \& Santos-Rosa, F. (2001). Un estudio piloto de los antecedentes disposicionales y contextuales relacionados con el estado de flow en competición. Comunicacion presentada en el II Congreso de Ciencias de la ActividadFisica y el Deporte. Valencia, España.

Csikszentmihalyi, M. (1975). Beyond boredom and anxiety. San Francisco: Jossey- Bass.

Csikszentmihalyi, M. (1998). Aprender a fluir. Barcelona: Kairós.

Custodero, L. A. (1998). Observing flow in young children's music learning. General Music Today, 12(1), 21-27.

Custodero, L. A. (2002). Seeking challenge, finding skill: Flow experience and music education. Arts Education Policy Review, 103(3), 3-9.

Custodero, L. A. (2005). Observable indicators of flow experience: A developmental perspective on musical engagement in young children from infancy to school age. Music Education Research, 7(2), 185-209.

Fabra-Brell, E., \& Romero-Naranjo, F. J. (2017). Social competence between equals through body percussion according to method BAPNE in secondary students. Procedia-Social and Behavioral Sciences, 237, 829-836.

García-Calvo, T., Cervelló, E., Jiménez, R., \& Santos-Rosa, F. J. (2005). Propiedades psicométricas de la Flow State Scale y la Dispositional Flow Scale, en sujetos españoles practicantes de actividades físicodeportivas.

García-Calvo, T., Jiménez, R., Santos-Rosa, F. J., Reina, R., \& Cervelló, E. (2008). Psychometric properties of the Spanish version of the Flow State Scale. Spanish Journal of Psychology, 11, 660-669. 
Estudio piloto de variables socio-emocionales, ansiedad y flow en alumnos de grado profesional de música mediante actividades BAPNE

Laura Moral Bofill, Francisco Vicedo Molla y Francisco Javier Romero Naranjo

Gardner, H. (1987). Estructuras de la mente: La teoría de las inteligencias múltiples. México: Fondo de Cultura Económica.

Gardner, H. (1995). Inteligencias múltiples. La teoría en la práctica. Barcelona: Espasa Libros.

Gardner, H. (2010). La inteligencia reformulada. Las inteligencias múltiples en el S. XXI. Barcelona: Paidós Ibérica.

Jackson, S. A., Kimiecik, J. C., Ford, S., \& Marsh, H. W. (1998). Psychological correlates of flow im sport. Journal of Sport and Exercise Psychology, 20, 358-378.

Jackson, S. A., \& Marsh, H. W. (1996). Development and validation os scale to measure optimal experience: The flow state scale. Journal of Sport and Exercise Psychology, 18, 17-35.

Márquez, A. C., \& Tresserra, M. P. (2018). 10 ideas clave. Neurociencia y educación: Aportaciones para el aula (Vol. 27). Barcelona: Graó.

Mora, F. (2017). Neuroeducación. Madrid: Alianza Editorial.

Moral-Bofill, L., Romero Naranjo, F. J., Albiar-Aliaga, E., \& Cid-Lamas, J. A. (2015). The BAPNE Method as a School Intervention and Support Strategy to Improve the School Environment and Contribute to Socioemotional Learning (SEL). International Journal of Innovation and Research in Educational Sciences (IJIRES), 2(6), 450-456.

Romero-Naranjo, F. J. (2013). Science \& art of body percussion: A review. Journal of human sport and exercise. 8(2), 442-457. Retrieved from http://www.jhse.ua.es/jhse/ article/view/556/749

Romero-Naranjo, F. J. (2017a). Bapne Method. Barcelona: Body Music Body Percussion Press.

Romero-Naranjo, F. J. (2017b). Bodypercussion. Programación didáctica. Alicante: Romero-Naranjo.

Romero-Naranjo, F. J. (2018). Bodypercussion. Basic. Barcelona: Body Music Body Percussion Press.

Silva, F., \& Martorell, M. C. (1982). La Batería de Socialización. Valencia: Promolibro.

Silva, F., \& Martorell, M. C. (1983). BAS-1 y 2 Batería de Socialización (para profesores y padres). Madrid: TEA.

Silva, M., \& Martorell, M. C. (2009). BAS, Batería de Socialización (BAS-3). Madrid: TEA.

Silva, F., Martorell, M. C., \& Clemente, A. (1985). Evaluación de la socialización y sus relaciones con inteligencia y dimensiones de personalidad en los niños mayores y adolescentes. Evaluación Psicológica/Psychological Assesment, 1, 241-266.

Spielberger, C. D., Gorsuch, R. L., \& Lushene, R. E. (1970). Manual for the state-trait anxiety inventory.

Spielberger, C. D. (1966). Theory and research on anxiety. Anxiety and behavior, 1, 3-20.

Spielberger, C. D. (1972a). Conceptual and methodological issues in anxiety research. Anxiety: Current trends in theory and research, 1.

Spielberger, C. D. (1972b). Conceptual and methodological issues in anxiety research. Anxiety: Current trends in theory and research, 2, 481-493.

Spielberger, C. D. (1989). Cuestionario de Autoevaluación Ansiedad Estado-Rasgo en niños, STAIC. Madrid: TEA Ediciones. 